\title{
Trabectedin for reversing platinum resistance and resensitization to platinum in patients with recurrent ovarian cancer
}

\author{
Antonio Casado*,1, Hector R Callata ${ }^{1}$, Aranzazu Manzano ${ }^{1}$, Gloria Marquina ${ }^{1}$, Teresa \\ Alonso1, Pablo Gajate1, Miguel Sotelo', Santiago Cabezas ${ }^{1}$, Cristina Fernández² \& Eduardo \\ Díaz-Rubio ${ }^{1}$ \\ ${ }^{1}$ Department of Medical Oncology, University Hospital San Carlos, Madrid, Spain \\ ${ }^{2}$ Department of Public Health \& Preventive Medicine, University Hospital San Carlos, Madrid, Spain \\ *Author for correspondence: Tel.: +34 913303 702; Fax: +34 913303 544; antonio.casado@salud.madrid.org
}

\begin{abstract}
Aims: We evaluated trabectedin in patients with platinum-resistant/refractory and partially platinumsensitive recurrent ovarian cancer and the outcomes after reintroduction of platinum. Methods: Twentyseven patients (platinum-resistant/refractory $n=24 / P P S ; n=3$ ) treated with trabectedin were retrospectively analyzed. Results: Trabectedin resulted in an objective response rate (ORR) of $18.2 \%$ with a $59.1 \%$ of disease control rate (ORR plus stable disease). The median progression-free and overall survival were 3.0 and 21.3 months, respectively. Subsequently, 17 patients were retreated with platinum and yield an ORR of $41.2 \%$ and DCR of $47.0 \%$. The median progression-free and overall survival after platinum rechallenge were 5.0 and 14.7 months, respectively. Conclusion: Our results suggest that trabectedin may contribute to resensitize tumor cells to platinum rechallenge.
\end{abstract}

First draft submitted: 23 July 2018; Accepted for publication: 24 September 2018; Published online: 22 November 2018

Keywords: ovarian cancer $\bullet$ platinum rechallenge $\bullet$ platinum-resistant/refractory • resensitization $\bullet$ trabectedin

Ovarian cancer is the leading cause of death from gynecological malignancies and the seventh leading cause of cancer-related death among women in the EU in the period 1970-2003 [1]. Early stage ovarian carcinoma is generally asymptomatic, thus often resulting in a late diagnosis with approximately $75 \%$ of all the cases presenting with an advanced stage associated with poor outcome [2]. Despite of high response rates to front-line treatment for advanced disease with cytoreductive surgical debulking followed by platinum/taxane-based chemotherapy (up to $80 \%$ ), most patients with advanced disease relapse within 2 years from diagnosis [3].

Recurrent ovarian cancer (ROC) is not a curable disease; thus, the principal objective of salvage treatments is to prolong survival, alleviate cancer-related symptoms and improve quality of life [4,5]. The usual treatment approach in relapsed patients is determined by their response to the first-line platinum-based chemotherapy, as measured by the platinum-free interval (PFI) [6]. Patients with platinum-sensitive disease, defined as a PFI $\geq 6$ months, are usually re-treated with platinum-containing regimens, yielding response rates ranging from 30-60\% [7]. Among platinum-sensitive patients, patients with partially platinum-sensitive disease (PPS), who experience disease relapse from 6-12 months after front-line platinum-based therapy, typically obtain substantially lower response rate to platinum re-challenge [8-10]. On the other side, no best postprogression treatment at relapse is agreed on for chemoresistant women whose disease progresses while receiving initial platinum-based treatment or within 4 weeks of last platinum dose (platinum-refractory) and those experiencing progressive disease (PD) within 6 months (PFI $<6$ months) of platinum-based therapy in the primary setting (i.e., platinum-resistant) and thus, represents a therapeutic challenge [6]. Taking into consideration that the likelihood of response to re-treatment with platinumbased treatment in patients with platinum-resistant/refractory (PRR) ROC is low, nonplatinum monotherapy is the preferred treatment for those patients. A variety of single agents, including paclitaxel, etoposide, gemcitabine, topotecan, pemetrexed and pegylated liposomal doxorubicin (PLD) have shown limited activity in patients with

Future Medicine 
PRR disease with an objective response rate (ORR) usually between 6-25\%, median progression-free survival (PFS) ranging from 3-5 months and median survival time from 11-13 months [11-17].

Trabectedin (Yondelis ${ }^{\circledR}$ ) is the first anticancer marine drug to be approved by the EU and many other countries worldwide. Results from a Phase III randomized study conducted in 672 patients with platinum-sensitive and platinum-resistant ROC (OVA-301; NCT00113607) showed a clinically relevant benefit for the combination of trabectedin plus PLD as compared with PLD alone $[18,19]$. Trabectedin has a unique multifaceted mechanism of action that involves transcription regulation and DNA repair systems, including transcription-coupled nucleotide excision repair and homologous recombination as the main hallmarks of its antiproliferative activity [20]. In addition to direct growth inhibition, trabectedin at therapeutic concentrations has selective anti-inflammatory and immunomodulatory properties due to the inhibition of production of factors that promote tumor growth, angiogenesis and metastasis [21-23]. Recently, it has been reported that cancer cells that have become less sensitive or resistant to trabectedin, following repeated exposures to trabectedin, become much more sensitized to next platinumbased chemotherapy [24]. In addition, two analyses of overall survival (OS) carried out in all platinum-sensitivity subsets of patients treated in the OVA-301 study showed that the treatment with trabectedin plus PLD prior to subsequent platinum retreatment results in at least 6-month favorable trend toward longer OS compared with PLD alone $[25,26]$. Those results have provided further supportive clinical evidence for the sequential combination of trabectedin and platinum. Based on these observations, we retrospectively analyzed data from heavily pretreated patients with PRR and PPS ROC who had been treated with trabectedin and subsequent platinum at our institution to evaluate, if trabectedin may play a role in reverting platinum resistance and resensitization to further platinum.

\section{Methods}

\section{Study design}

For this retrospective analysis, patient attendance records and the institutional chemotherapy database were searched for all patients commencing chemotherapy with trabectedin for advanced ROC, between January 2003 and March 2013 at the University Hospital San Carlos in Madrid, Spain. Eligible patients were patients $\geq 18$ years old with unresectable advanced or metastatic, histologically proven recurrent epithelial ovarian carcinoma who had experienced either relapse or disease progression. All patients were duly informed about treatment and signed informed consents were obtained from all study participants before any treatment. In addition, all procedures performed in studies involving human participants were in accordance with the ethical standards of the institutional and/or national research committee and with the 1964 Helsinki declaration and its later amendments or comparable ethical standards.

\section{Treatments}

Trabectedin was administered as a 3-h intravenous infusion every 3 weeks. The initial dose of trabectedin ranged between $1.1 \mathrm{mg} / \mathrm{m}^{2}$ body surface area, which is the recommended dose for trabectedin when combined with PLD [18] and $1.5 \mathrm{mg} / \mathrm{m}^{2}$. Antiemetic and hepatoprotective prophylaxis with dexamethasone $20 \mathrm{mg}$ intravenously $30 \mathrm{~min}$ prior to trabectedin and serotonin antagonists were considered mandatory for all patients. In addition, antiemesis prophylaxis included corticosteroid medication with dexamethasone or prednisone pre- and postchemotherapy. Treatment with trabectedin was discontinued at progression or for other reasons such as unacceptable toxicity, investigator decision or consent withdrawal.

\section{Study evaluations}

Tumor response was assessed at screening and every 12 weeks thereafter, according to the standard response evaluation criteria in solid tumors v.1.0 and v.1.1 [27,28]. Radiological efficacy assessments of trabectedin and posttrabectedin treatments were conducted in an all-evaluable patient population. The disease control rate (DCR) was defined as the percentage of patients with a complete response (CR) plus partial response (PR) plus stable disease (SD). Duration of response was defined as the time from the start of treatment until documentation of PD. For the time-to-event analyses, as time-to-progression (TTP), PFS and OS, the Kaplan-Meier method was used to estimate fixed time points. The TTP, PFS and OS analyses following trabectedin and post-trabectedin platinumbased treatment were defined as the time interval from the first administration of trabectedin or subsequent platinum treatment to disease progression for TTP or until disease progression, death or lost to follow-up for PFS, whereas OS was defined as the time between the start of trabectedin or subsequent platinum administration and patient death from any cause or last follow-up. Moreover, PFI was defined as the interval from the last date 
Table 1. Patient and disease characteristics at baseline.

\begin{tabular}{|c|c|}
\hline Patients & $(n=27) n(\%)$ \\
\hline \multicolumn{2}{|c|}{ Age at treatment with trabectedin (years): } \\
\hline - Median (range) & $63(45-81)$ \\
\hline \multicolumn{2}{|l|}{ Platinum sensitivity: } \\
\hline$-P R R$ & $24(88.9)$ \\
\hline - PPS & $3(11.1)$ \\
\hline \multicolumn{2}{|c|}{ Number of prior chemotherapy lines: } \\
\hline - Median (range) & $5(1-9)$ \\
\hline-1 & $3(11.1)$ \\
\hline-4 & $5(18.5)$ \\
\hline$-\geq 5$ & $19(70.4)$ \\
\hline \multicolumn{2}{|l|}{ ECOG PS: } \\
\hline-0 & $11(40.7)$ \\
\hline-1 & $12(44.4)$ \\
\hline$-\geq 2$ & $4(14.8)$ \\
\hline \multicolumn{2}{|l|}{ Histology: } \\
\hline - Serous-papillary & $18(66.6)$ \\
\hline - Clear-cell & $3(11.1)$ \\
\hline - Endometrioid & $2(7.4)$ \\
\hline - Undifferentiated & $2(7.4)$ \\
\hline - Mucinous & $2(7.4)$ \\
\hline
\end{tabular}

of platinum-based therapy until documented disease progression. For subsequent platinum-based chemotherapy, administered after disease progression and discontinuation of trabectedin, the tumor response and the outcomes of time-to-event assessments were also recorded.

\section{Results}

\section{Patient characteristics}

This analysis included a total of 27 patients with PD at the time of trabectedin initiation. Overall, 24 patients were considered resistant/refractory to platinum (88.9\%), whereas three patients had PPS disease (11\%) following the initial treatment with carboplatin and paclitaxel. Patients and tumor characteristics are summarized in Table 1. At the time of treatment with trabectedin patients had a median age of 63 years (range: $45-81$ years) and most had serous-papillary carcinoma $(\mathrm{n}=18 ; 66.6 \%)$ and a good Eastern Cooperative Oncology Group performance status score of $0 / 1$ was recorded in $23(85.2 \%)$ patients. Patients were pretreated with a median of five lines of chemotherapy (range: 1-9 lines) prior to trabectedin administration with 19 of them (70.4\%) who were exposed to more than five lines of prior chemotherapy. Several nonplatinum-based chemotherapies were intercalated between front-line treatment with platinum and before the treatment with trabectedin. Most patients received weekly topotecan $(n=17)$, bevacizumab $(n=13)$ or PLD $(n=11)$, whereas oral chronic etoposide, gemcitabine and paclitaxel were given to four, three and one patient, respectively.

\section{Response to trabectedin treatment}

Patients received a median of 3.5 trabectedin cycles per patient (range: 1-14 cycles) with no signs of cumulative toxicities. Overall, 14 patients received $<$ four cycles $(51.8 \%)$, four patients received 4-5 cycles (14.8\%) and nine patients $>$ six cycles $(33.3 \%)$ of trabectedin. The most commonly used trabectedin dose was $1.3 \mathrm{mg} / \mathrm{m}^{2}(\mathrm{n}=20$; $74.1 \%$ ), followed by 1.1 and $1.5 \mathrm{mg} / \mathrm{m}^{2}$ administered to six $(22.2 \%)$ and one $(3.7 \%)$ patients, respectively. The median interval between the last platinum treatment and the first trabectedin dose was 5 months (range: 1.0-18.0 months) with an interquartile range of 1.3-9.0 months.

At time of analysis, 22 of 27 patients $(81.5 \%)$ were assessable for response as two patients early progressed and died and three patients were considered not evaluable due to clinical deterioration. Regarding the overall 


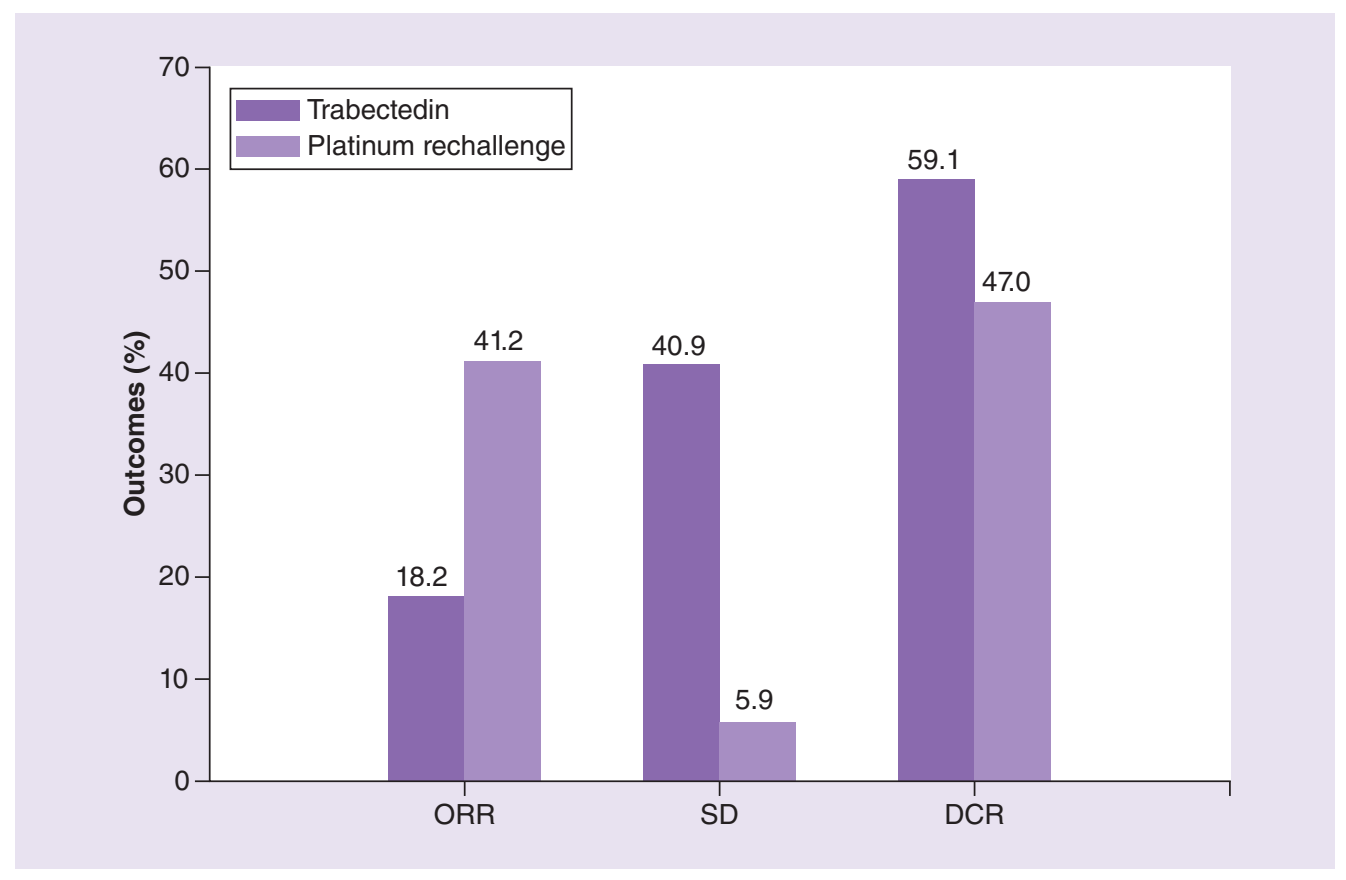

Figure 1. Responses to trabectedin and subsequent platinum-based treatment. DCR: Disease control rate; ORR: Objective response rate; SD: Stable disease.

trabectedin activity, one patient (4.5\%) had a CR and three patients (13.6\%) obtained PR reaching the ORR of $18.2 \%$ with a median duration of 16.5 weeks (range: 5.9-44.4 weeks). Moreover, nine (40.9\%) patients had SD as a best result with a median duration of 5.8 months (range: $4.1-9$ months) for a DCR of $59.1 \%$ (Figure 1). Nine $(40.9 \%)$ patients experienced disease progression during the treatment with trabectedin. Treatment with trabectedin resulted in a median PFS of 3.0 months (95\% CI: 2.2-3.7) and median OS of 21.3 months (95\% CI: 15.1-27.5) (Figure 2).

\section{Response to platinum rechallenge}

Seventeen patients who progressed after a median of six cycles of trabectedin and with no contraindications or morbidities to be rechallenged with platinum salts, were eligible to be retreated with subsequent platinum-based chemotherapy. Five patients were not rechallenged with platinum after trabectedin owing to a rapid deterioration and death, two patients had bowel obstruction, one patient refused further treatment with platinum, one had renal insufficiency and another patient had a severe hypersensitivity reaction to carboplatin that hindered her subsequent treatments with platinum.

After the median duration of time between the last trabectedin administration and the first subsequent platinum dose of 2.5 months (range: 1-5 months), eligible patients were rechallenged with a median of six cycles (range: 1-10 cycles) of platinum-based chemotherapy. The most frequently used combination was carboplatin plus paclitaxel in eight patients (pretreated with a median of four prior chemotherapy lines; range: 1-8) followed by carboplatin plus paclitaxel and bevacizumab in four, carboplatin plus gemcitabine in two patients and carboplatin monotherapy, carboplatin plus PLD and oxaliplatin with paclitaxel and bevacizumab in one patient each. Seven patients had confirmed a PR according to response evaluation criteria in solid tumors giving an ORR of $41.2 \%$. Of note, the ORR was retained in patients pretreated with $\geq 5$ prior platinum-based lines. Additionally, one patient (5.9\%) had SD for a DCR of $47.0 \%$ (Figure 1). Analysis of time-to-event end points following platinum rechallenge resulted in a median PFS of 5.0 months (95\% CI: 4.0-5.9 months) and a median OS of 14.7 months (95\% CI: 5.7-23.6 months) in this heavily pretreated-patient population (Figure 3).

An analysis of the patients who were treated with trabectedin and achieved an ORR, SD or disease progression showed that the patient who had a CR with trabectedin did not respond to subsequent carboplatin treatment, whereas among the three patients who achieved PR with trabectedin one had PR to subsequent carboplatin, one patient progressed and another was not rechallenged with platinum. Among those who obtained SD with 

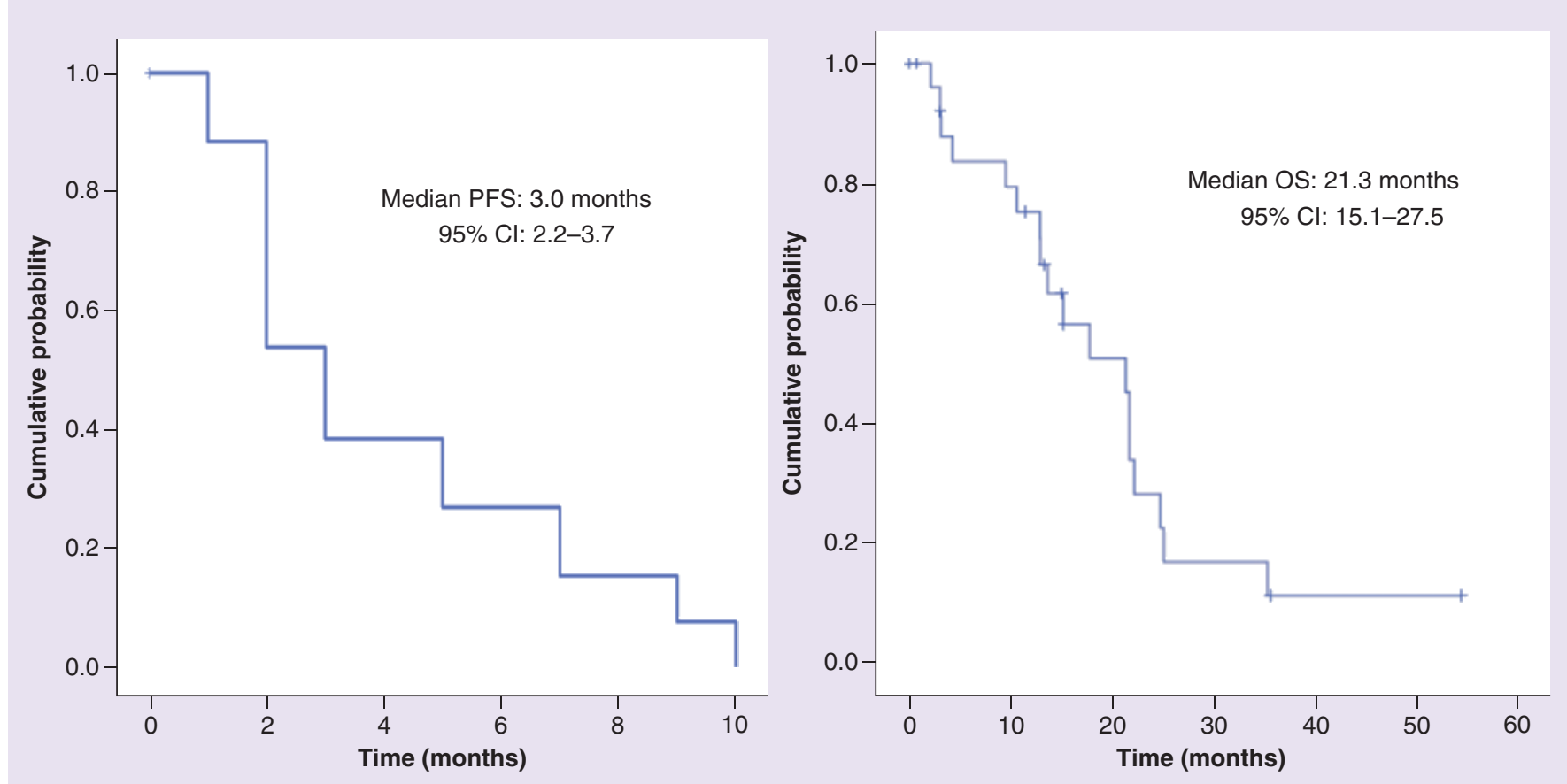

Figure 2. Median progression-free survival and overall survival following the treatment with trabectedin.
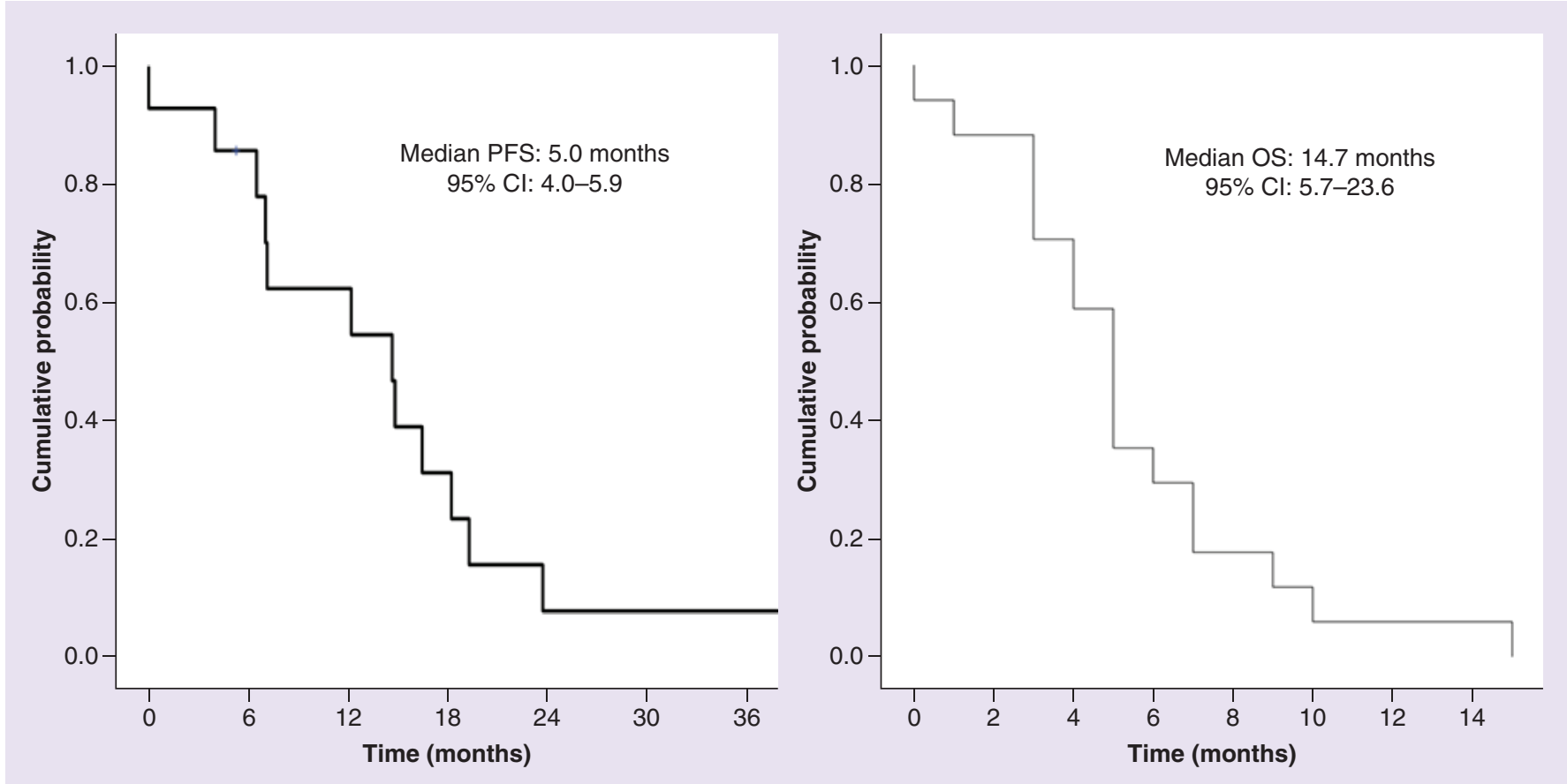

Figure 3. Median progression-free survival and overall survival after trabectedin treatment and platinum rechallenge.

trabectedin, five patients attained PR after subsequent platinum, two patients progressed and another patient had SD (one of those patients did not receive subsequent platinum). Finally, among patients who progressed with trabectedin, one had a PR, two obtained SD and three patients also progressed after the treatment with subsequent platinum. 


\section{Discussion}

Patients with PRR ROC have a dismal prognosis with a mean life expectancy of approximately 12 months despite treatment with salvage chemotherapy. For this group of patients, therapeutic options are limited and usually consist in nonplatinum agents. This retrospective analysis aimed to evaluate the efficacy of trabectedin monotherapy and the outcomes after reintroduction of a platinum-based treatment. To date, three Phase II studies evaluating the efficacy of single agent trabectedin in the treatment of ROC demonstrated response rates of 39-43\% [29-32]. The results of an integrated pooled analysis of Phase II studies have confirmed that trabectedin as single agent is an active treatment in ROC as reported an ORR for the whole pooled population of $26 \%$ and a median OS of 17.1 months [32]. In addition, one prospective study in patients with $B R C A$ patients and another retrospective multicenter study showed similar response rates $(27.5-40 \%)$ [33,34]. The results of this single institution series suggest that trabectedin is an effective treatment that provides clinically meaningful benefits to heavily pretreated patients with PRR/PPS ROC (e.g., ORR: 18.2\%; median OS: 21.3 months) and favorably compare with those observed in patients with platinum-resistant ROC and treated with trabectedin as the second/third-line monotherapy in Phase II studies [29-32] and are comparable with those seen with other nonplatinum agents [11-17]. We should point out that due to a worldwide shortage of PLD, caused by an 18-month halt in production from 2011 to 2013 , single agent trabectedin was proposed as an alternative to our patients with ROC. The outcomes from our analysis were achieved in a population that had been heavily treated with a median of five lines of chemotherapy (range: 1-9 lines) and with $70 \%$ patients pretreated with $\geq 5$ prior regimens before trabectedin administration. Arguably, advances in supportive care could explain, at least partly, the improved outcomes in our series. Although, due to its retrospective nature, this study was not designed to evaluate the safety of trabectedin, the patients received a median of 3.5 cycles of trabectedin, suggesting an acceptable safety profile that allowed prolonged treatment (i.e., up to 14 cycles), being consistent with previous reports of trabectedin safety [35].

Studies in human ovarian cancer cells and patients with platinum-resistant ovarian cancer have reported that primary or secondary (acquired) resistance to platinum could be a variable phenotype over time, which could be considered as an unstable, inducible, multifactorial and reversible phenomenon after a drug-free interval and/or sequential treatment with nonplatinum drugs [36-38]. Up to date trabectedin is the only compound for which convincing experimental evidence of the platinum-restoration mechanism is available [39]. Noteworthy, the results of the analysis among patients who obtained disease progression as a best response with trabectedin and were rechallenged with platinum suggest that a response to trabectedin is not requested to potentially obtain a benefit from the next rechallenge with platinum-based therapies. In contrast with what has been reported for other DNAinteracting drugs like platinum salts, which are more effective against cells with defects in nucleotide excision repair mechanism (NER) [40,41], trabectedin efficacy is linked to an efficient transcription-coupled NER and deficient homologous recombination repair activity in the targeted tumor cells [20,33,42-44]. Several preclinical data have indicated that trabectedin treatment enhances the collateral sensitivity to subsequent treatments with platinum drugs. Recent in vitro and in vivo studies evaluating trabectedin-resistant cell lines from ovarian carcinoma and myxoid liposarcoma have confirmed that resistance to trabectedin is associated with the loss of NER function, with a consequent increased sensitivity to platinum drugs [24]. Thus, these data suggested that cancer cells that have become less sensitive or resistant to trabectedin after repeated exposures to trabectedin become much more sensitized to next platinum-based chemotherapy. Those data have provided a clear molecular rationale for the efficacy of the sequential use of trabectedin and platinum drugs. Importantly, those results seem to translate well into clinically useful approaches considering the results from two post hoc, exploratory analyses of OS performed in patients according to platinum-sensitivity categories [25] and separately in patients with platinum-resistant disease [26], who were subsequently retreated with platinum-based chemotherapy after completing either trabectedin plus PLD or PLD treatment in OVA-301 trial. In all subsets of patients, the treatment with trabectedin plus PLD prior to subsequent third-line platinum retreatment resulted in an at least 6-month favorable trend toward longer OS compared with PLD alone. Significantly longer OS was recorded in patients with PPS disease with a notable improvement of 9 months in median OS (median OS: 27.7 vs 18.7 months; $p<0.0153$ ) [25], whereas a 6.7-month improvement in median OS and a $38 \%$ decrease in the risk of death was observed in patients with platinum-resistant disease (22.2 vs 15.5 months; hazard ratio: $0.62,95 \%$ CI: $0.36-1.06 ; \mathrm{p}=0.078$ ). Of note, the median OS reported in the cohort of patients with platinum-resistant ROC treated with trabectedin plus PLD in the OVA-301 trial (22.2 months) is very similar to that observed in this analysis (21.3 months) [26]. 
Apart from the limited number of patients and the retrospective nature of this study, the principal limitation of this study was the lack of the control platinum-based arm in order to demonstrate that trabectedin increases the likelihood of response to subsequent platinum over immediate platinum-based re-treatment. Although speculative, it could be also argued the hypothesis that in a less heavily pretreated patient population, the efficacy of trabectedin and the results of platinum rechallenging could have led to a more extensive benefit. Taking into account that no prospective data are available comparing trabectedin or trabectedin plus PLD with a platinum-based postprogression regimens, two prospective clinical trials are ongoing aiming to shed some more light on this issue. The results of this retrospective study provided the rationale for designing a Phase II clinical trial to prospectively evaluate the effect of reintroduction of platinum salts after trabectedin treatment in women with platinum-resistant ROC who have been pretreated with a maximum of four chemotherapy lines (No EudraCT: 2014-004020-21). Moreover, the ongoing academic Phase III INOVATYON (refers to: INternational OVArian cancer patients Treated with YONdelis) trial is aimed to demonstrate that the combination of trabectedin plus PLD prolongs OS over carboplatin plus PLD in patients with PPS ROC (ClinicalTrials.gov identifier: NCT01379989). Indeed, in both the trials, the expected improvement in survival could be not only related to intercalation of the combination of trabectedin plus PLD or trabectedin alone before subsequent platinum rechallenge but also due to a peculiar aspect of the mechanism of action of trabectedin that could enhance the efficacy of subsequent platinum-based therapies.

\section{Conclusion}

In conclusion, the results of this retrospective, real-life analysis suggest that sequential treatment with trabectedin might resensitize the tumor cells of patients with PRR and PPS ROC and improve the efficacy of subsequent platinum-based chemotherapy.

\section{Future perspective}

Although platinum-based drugs are regularly used in patients with platinum-sensitive disease, uncertainties abound in the treatment of women with PPS relapse and women unsuited for platinum rechallenge. Particularly the results from the Phase III OVA-301 study have called into question the paradigm of platinum as mainstay treatment for patients with platinum-sensitive relapse. The results of further prospective Phase II-III clinical studies and expanded access programs will probably definitely confirm the role of the non-platinum combination of trabectedin plus PLD (and/or trabectedin monotherapy) in relapsed ovarian cancer patients with a platinum-free interval (PFI) beyond 6 months. It will be particularly the case in patients who experience residual toxicities to platinum, have limited sensitivity to platinum (i.e., for patients with PPS disease with a PFI of 6-12 months), or have developed hypersensitivity reactions to platinum. Nevertheless, to date in the absence of randomized trials it is impossible to demonstrate the superiority of trabectedin plus PLD over standard platinum-based chemotherapy in the setting of platinum-sensitive recurrent disease. Notably, a Phase II clinical trial will prospectively evaluate the effect of re-introduction of platinum salts after trabectedin treatment in women with platinum-resistant ROC, whereas the ongoing academic Phase III INOVATYON trial is aimed to demonstrate that the combination of trabectedin plus PLD prolongs overall survival over carboplatin plus PLD in patients with PPS disease.

\section{Acknowledgements}

The authors would like to acknowledge Adnan Tanović (PharmaMar, SA) for providing writing and editorial assistance for the manuscript.

Financial \& competing interests disclosure

The authors have no relevant affiliations or financial involvement with any organization or entity with a financial interest in or financial conflict with the subject matter or materials discussed in the manuscript. This includes employment, consultancies, honoraria, stock ownership or options, expert testimony, grants or patents received or pending, or royalties. 
Open access

This work is licensed under theAttribution-NonCommercial-NoDerivatives 4.0 Unported License. To view a copy of this license, visit http://creativecommons.org/licenses/by-nc-nd/4.0/

\section{Executive summary}

- We retrospectively analyzed 27 heavily pretreated patients (24 with platinum-resistant/refractory [PRR]/three with partially platinum-sensitive [PPS] recurrent ovarian cancer [ROC]). Tumor response was assessed every 12 weeks according to Response Evaluation Criteria in Solid Tumors.

- This retrospective analysis was performed to better understand, if the treatment with trabectedin may increase the likelihood of response to subsequent platinum in patients with PRR and PPS ROC.

- Principal molecular rationale for the efficacy of such a sequential use lays in the fact that cancer cells, which have become less sensitive or resistant to trabectedin after repeated exposures to trabectedin, become much more sensitized to next platinum-based chemotherapy.

- Preclinical data have suggested that trabectedin enhances the collateral sensitivity to subsequent treatments with platinum salts by reverting platinum resistance and resensitization to platinum.

Main results

- Patients received a median of 3.5 trabectedin cycles per patient (range: 1-14 cycles) with no signs of cumulative toxicities.

- Among 22 patients $(81.5 \%)$ assessable for response with trabectedin one (4.5\%) had a complete response and three patients $(13.6 \%)$ obtained partial response reaching the objective response rate of $18.2 \%$. Nine other $(40.9 \%)$ patients achieved stable disease for a disease control rate of $59.1 \%$. Treatment with trabectedin resulted in a median progression-free survival of 3.0 months and median overall survival of 21.3 months.

- After a median time between the last trabectedin and the subsequent platinum dose of 2.5 months, 17 patients were retreated with platinum, seven of whom had a partial response giving an objective resonse rate of $41.2 \%$. Additionally, one patient (5.9\%) had stable disease for a disease control rate of $47.0 \%$. The median progression-free survival and overall survival after platinum rechallenge were 5.0 months and 14.7 months, respectively.

Conclusion

- The results of this retrospective, real-life analysis suggest that sequential treatment with trabectedin followed by subsequent platinum rechallenge might re-sensitize the tumor cells of heavily pretreated patients with PRR and PPS ROC.

- Prospective studies are ongoing to definitely establish the role of trabectedin and sequential treatments in women with platinum-resistant and PPS ROC.

\section{References}

Papers of special note have been highlighted as: $\bullet$ of interest; $\bullet \bullet$ of considerable interest

1. Jemal A, Bray F, Center MM, Ferlay J, Ward E, Forman D. Global cancer statistics. CA Cancer J. Clin. 61(2), 69-90 (2011).

2. Laurvick CL, Semmens JB. Trends and outcomes for women diagnosed with ovarian cancer in Australia. Aust. Fam. Physician 31(11), 1005-1011 (2002).

3. Harter P, Hilpert F, Mahner S, Heitz F, Pfisterer J, Du Bois A. Systemic therapy in recurrent ovarian cancer: current treatment options and new drugs. Expert Rev. Anticancer Ther. 10(1), 81-88 (2010).

4. Ledermann J, Raja FA, Fotopoulou C et al. Newly diagnosed and relapsed epithelial ovarian carcinoma: ESMO Clinical Practice Guidelines for diagnosis, treatment and follow-up. Ann. Oncol. 24(Suppl. 6), vi24-vi32 (2013).

5. Poveda A, Ray-Coquard I, Romero I, Lopez-Guerrero JA, Colombo N. Emerging treatment strategies in recurrent platinum-sensitive ovarian cancer: focus on trabectedin. Cancer Treat. Rev. 40(3), 366-375 (2014).

-• Outlines the therapeutic potential of new emerging approaches, particularly the role of nonplatinum therapy with trabectedin.

6. Friedlander M, Trimble E, Tinker A et al. Clinical trials in recurrent ovarian cancer. Int. J. Gynecol. Cancer 21(4), 771-775 (2011).

7. Hall M, Rustin G. Recurrent ovarian cancer: when and how to treat. Curr. Oncol. Rep. 13(6), 459-471 (2011).

8. Colombo N, Gore M. Treatment of recurrent ovarian cancer relapsing 6-12 months post platinum-based chemotherapy. Crit. Rev. Oncol. Hematol. 64(2), 129-138 (2007).

9. Kaye S. Management of partially platinum-sensitive relapsed ovarian cancer. Eur. J. Cancer 6(4), 16-21 (2008).

- Supports the hypothesis that the enhanced survival benefits in the partially platinum-sensitive subset of patients with recurrent ovarian cancer (ROC) might be due to an extended platinum-free interval (PFI) leading to longer survival with subsequent platinum.

10. Pfisterer J, Ledermann JA. Management of platinum-sensitive recurrent ovarian cancer. Semin. Oncol. 33(2 Suppl. 6), S12-S16 (2006). 
11. Peng LH, Chen XY, Wu TX. Topotecan for ovarian cancer. Cochrane Database Syst. Rev. 2, CD005589 (2008).

12. Gordon AN, Fleagle JT, Guthrie D, Parkin DE, Gore ME, Lacave AJ. Recurrent epithelial ovarian carcinoma: a randomized Phase III study of pegylated liposomal doxorubicin versus topotecan. J. Clin. Oncol. 19(14), 3312-3322 (2001).

13. Mutch DG, Orlando M, Goss T et al. Randomized Phase III trial of gemcitabine compared with pegylated liposomal doxorubicin in patients with platinum-resistant ovarian cancer. J. Clin. Oncol. 25(19), 2811-2818 (2007).

14. Markman M, Blessing J, Rubin SC, Connor J, Hanjani P, Waggoner S. Phase II trial of weekly paclitaxel (80 mg/m2) in platinum and paclitaxel-resistant ovarian and primary peritoneal cancers: a Gynecologic Oncology Group study. Gynecol. Oncol. 101(3), 436-440 (2006).

15. Rose PG, Blessing JA, Mayer AR, Homesley HD. Prolonged oral etoposide as second-line therapy for platinum-resistant and platinum-sensitive ovarian carcinoma: a Gynecologic Oncology Group study. J. Clin. Oncol. 16(2), 405-410 (1998).

16. Miller DS, Blessing JA, Krasner CN et al. Phase II evaluation of pemetrexed in the treatment of recurrent or persistent platinum-resistant ovarian or primary peritoneal carcinoma: a study of the Gynecologic Oncology Group. J. Clin. Oncol. 27(16), 2686-2691 (2009).

17. Lortholary A, Largillier R, Weber B et al. Weekly paclitaxel as a single agent or in combination with carboplatin or weekly topotecan in patients with resistant ovarian cancer: the CARTAXHY randomized Phase II trial from Groupe d'Investigateurs Nationaux pour l'Etude des Cancers Ovariens (GINECO). Ann. Oncol. 23(2), 346-352 (2012).

18. Monk BJ, Herzog TJ, Kaye SB et al. Trabectedin plus pegylated liposomal doxorubicin in recurrent ovarian cancer. J. Clin. Oncol. 28(19), 3107-3114 (2010).

-. The results from the pivotal randomized Phase III OVA-301 trial, which compared pegylated liposomal doxorubicin (PLD) with a combination of trabectedin plus PLD that included both patients with platinum-resistant (PFI $<6$ months) and platinum-sensitive (PFI $\geq 6$ months) ROC.

19. Monk BJ, Herzog TJ, Kaye SB et al. Trabectedin plus pegylated liposomal doxorubicin (PLD) versus PLD in recurrent ovarian cancer: overall survival analysis. Eur. J. Cancer 48(15), 2361-2368 (2012).

20. D'Incalci M, Galmarini CM. A review of trabectedin (ET-743): a unique mechanism of action. Mol. Cancer Ther. 9(8), 2157-2163 (2010).

- Demonstrates that trabectedin selectively depletes mononuclear phagocytes, including tumor-associated macrophages, in tumor patients who receive trabectedin-based therapy. This represents a key component of the antitumor activity of trabectedin.

21. Allavena P, Signorelli M, Chieppa M et al. Anti-inflammatory properties of the novel antitumor agent yondelis (trabectedin): inhibition of macrophage differentiation and cytokine production. Cancer Res. 65(7), 2964-2971 (2005).

22. Allavena P, Germano G, Belgiovine C, D’Incalci M, Mantovani A. Trabectedin: a drug from the sea that strikes tumor-associated macrophages. Oncoimmunology 2(6), e24614 (2013).

23. Germano G, Frapolli R, Belgiovine $\mathrm{C}$ et al. Role of macrophage targeting in the antitumor activity of trabectedin. Cancer Cell 23(2), 249-262 (2013).

24. Colmegna B, Uboldi S, Frapolli R et al. Increased sensitivity to platinum drugs of cancer cells with acquired resistance to trabectedin. $B r$. J. Cancer 113(12), 1687-1693 (2015).

-• Reports that resistance to trabectedin is associated with the loss of nucleotide excision repair mechanism function, with a consequent increased sensitivity to platinum drugs, which provides the rational for sequential use of these drugs in patients who have acquired resistance to trabectedin.

25. Colombo N, Tanovic A, Fernández C, Casado Herraez A. Trabectedin plus pegylated liposomal doxorubicin (PLD) prior to subsequent platinum-based chemotherapy in recurrent ovarian cancer (ROC): results form OVA-301 follow-up. Int. J. Gynecol. Cancer. 24(9 Suppl. 4), 452-453 Abstract 1242 (2014). Presented at: 15 $5^{\text {th }}$ Biennial Meeting of the International Gynecologic Cancer Society (IGCS). Melbourne, Victoria, Australia, 8-11 November 2014.

26. Colombo N, Casado A, Fernandez C, Vergote I. Trabectedin plus pegylated liposomal doxorubicin (PLD) prior to subsequent platinum chemotherapy in patients with platinum-resistant (PR) recurrent ovarian cancer (ROC): results from OVA-301 follow-up. J. Clin. Oncol. 32 (5 Suppl.), Abstract 5551 (2014). Presented at: $50^{\text {th }}$ Annual Meeting of the American Society of Clinical Oncology (ASCO). Chicago, IL, USA, 30 May-3 June 2014.

-• Hypothesizes that the intercalation with trabectedin plus PLD prior to subsequent platinum retreatment may resensitize patients with platinum-resistant ROC and to allow further platinum retreatment, which ultimately leads to longer survival.

27. Therasse P, Arbuck SG, Eisenhauer EA et al. New guidelines to evaluate the response to treatment in solid tumors. European Organization for Research and Treatment of Cancer, National Cancer Institute of the United States, National Cancer Institute of Canada. J. Natl Cancer Inst. 92(3), 205-216 (2000).

28. Eisenhauer EA, Therasse P, Bogaerts J et al. New response evaluation criteria in solid tumours: revised RECIST guideline (version 1.1). Eur. J. Cancer 45(2), 228-247 (2009).

29. Sessa C, De Braud F, Perotti A et al. Trabectedin for women with ovarian carcinoma after treatment with platinum and taxanes fails. J. Clin. Oncol. 23(9), 1867-1874 (2005). 
30. Krasner CN, McMeekin DS, Chan S et al. A Phase II study of trabectedin single agent in patients with recurrent ovarian cancer previously treated with platinum-based regimens. Br. J. Cancer 17(19), 1618-1624 (2007).

31. Del Campo JM, Roszak A, Bidzinski M et al. Phase II randomized study of trabectedin given as two different every 3 weeks dose schedules $\left(1.5 \mathrm{mg} / \mathrm{m}^{2} 24 \mathrm{~h}\right.$ or $\left.1.3 \mathrm{mg} / \mathrm{m}^{2} 3 \mathrm{~h}\right)$ to patients with relapsed, platinum-sensitive, advanced ovarian cancer. Ann. Oncol. 20(11), 1794-1802 (2009).

32. Del Campo JM, Sessa C, Krasner CN et al. Trabectedin as single agent in relapsed advanced ovarian cancer: results from a retrospective pooled analysis of three Phase II trials. Med. Oncol. 30(1), 435 (2013).

33. Lorusso D, Scambia G, Pignata $S$ et al. Prospective Phase II trial of trabectedin in BRCA-mutated and/or BRCAness phenotype recurrent ovarian cancer patients: the MITO 15 trial. Ann. Oncol. 27(3), 487-493 (2016).

34. Ferrandina G, Salutari V, Vincenzi B et al. Trabectedin as single agent in the salvage treatment of heavily treated ovarian cancer patients: a retrospective, multicenter study. Gynecol. Oncol. 130(3), 505-510 (2013).

35. Le Cesne A, Yovine A, Blay JY et al. A retrospective pooled analysis of trabectedin safety in 1132 patients with solid tumors treated in Phase II clinical trials. Invest. New Drugs 30(3), 1193-1202 (2012).

36. Horowitz NS, Hua J, Gibb RK, Mutch DG, Herzog TJ. The role of topotecan for extending the platinum-free interval in recurrent ovarian cancer: an in vitro model. Gynecol. Oncol. 94(1), 67-73 (2004).

37. Rose PG, Mossbruger K, Fusco N, Smrekar M, Eaton S, Rodriguez M. Gemcitabine reverses cisplatin resistance: demonstration of activity in platinum- and multidrug-resistant ovarian and peritoneal carcinoma. Gynecol. Oncol. 88(1), 17-21 (2003).

38. Kavanagh J, Tresukosol D, Edwards $\mathrm{C}$ et al. Carboplatin reinduction after taxane in patients with platinum-refractory epithelial ovarian cancer. J. Clin. Oncol. 13(7), 1584-1588 (1995).

39. Tomao F, D'Incalci M, Biagioli E, Peccatori FA, Colombo N. Restoring platinum sensitivity in recurrent ovarian cancer by extending the platinum-free interval: myth or reality? Cancer 123(18), 3450-3459 (2017).

40. Arora S, Kothandapani A, Tillison K, Kalman-Maltese V, Patrick SM. Downregulation of XPF-ERCC1 enhances cisplatin efficacy in cancer cells. DNA Repair 9(7), 745-753 (2010).

41. Colmegna B, Uboldi S, Erba E, D’Incalci M. Resistance to minor groove binders. Drug Discov. Today Technol. 11, 73-79 (2014).

42. Schoffski P, Taron M, Jimeno J et al. Predictive impact of DNA repair functionality on clinical outcome of advanced sarcoma patients treated with trabectedin: a retrospective multicentric study. Eur. J. Cancer 47(7), 1006-1012 (2011).

43. Monk BJ, Ghatage P, Parekh T et al. Effect of BRCA1 and XPG mutations on treatment response to trabectedin and pegylated liposomal doxorubicin in patients with advanced ovarian cancer: exploratory analysis of the Phase 3 OVA-301 study. Ann. Oncol. 26(5), 914-920 (2015).

44. Tavecchio M, Natoli C, Ubezio P, Erba E, D'Incalci M. Dynamics of cell cycle phase perturbations by trabectedin (ET-743) in nucleotide excision repair (NER)-deficient and NER-proficient cells, unraveled by a novel mathematical simulation approach. Cell Prolif. 40(6), 885-904 (2007). 